NONCOMMUTATIVE HARMONIC ANALYSIS

WITH APPLICATIONS TO PROBABILITY III

BANACH CENTER PUBLICATIONS, VOLUME 96

INSTITUTE OF MATHEMATICS

POLISH ACADEMY OF SCIENCES

WARSZAWA 2012

\title{
QUANTUM DETAILED BALANCE CONDITIONS WITH TIME REVERSAL: THE FINITE-DIMENSIONAL CASE
}

\author{
FRANCO FAGNOLA \\ Dipartimento di Matematica, Politecnico di Milano \\ Piazza Leonardo da Vinci 32, I-20133 Milano, Italy \\ e-mail: franco.fagnola@polimi.it \\ VERONICA UMANITÀ \\ Dipartimento di Matematica, Università di Genova \\ Via Dodecaneso 35, I-16146 Genova, Italy \\ e-mail:veronica.umanita@fastwebnet.it
}

\begin{abstract}
We classify generators of quantum Markov semigroups $\mathcal{T}$ on $\mathcal{B}(\mathrm{h})$, with $\mathrm{h}$ finitedimensional and with a faithful normal invariant state $\rho$ satisfying the standard quantum detailed balance condition with an anti-unitary time reversal $\theta$ commuting with $\rho$, namely $\operatorname{tr}\left(\rho^{1 / 2} x \rho^{1 / 2} \mathcal{T}_{t}(y)\right)=\operatorname{tr}\left(\rho^{1 / 2} \theta y^{*} \theta \rho^{1 / 2} \mathcal{T}_{t}\left(\theta x^{*} \theta\right)\right)$ for all $x, y \in \mathcal{B}(\mathrm{h})$ and $t \geq 0$.

Our results also show that it is possible to find a standard form for the operators in the Lindblad representation of the generators extending the standard form of generators of quantum Markov semigroups satisfying the usual quantum detailed balance condition with non-symmetric multiplications $x \mapsto \rho^{s} x \rho^{1-s}(s \in[0,1], s \neq 1 / 2)$ whose generators must commute with the modular group associated with $\rho$. This supports our conclusion that the most appropriate noncommutative version of the classical detailed balance condition is the above standard quantum detailed balance condition with an anti-unitary time reversal.
\end{abstract}

1. Introduction. A Markov semigroup on a commutative algebra satisfies the classical detailed balance condition, also called reversibility, when it is symmetric in the $L^{2}$ space of an invariant measure.

Several non-commutative versions have been proposed. The best known one, for a norm continuous Quantum Markov Semigroup (QMS) $\mathcal{T}$ on the algebra $\mathcal{B}(\mathrm{h})$ of linear

2010 Mathematics Subject Classification: 46L55, 47D06, 47D07, 82B10, 82C10, 81S25.

Key words and phrases: Quantum detailed balance, quantum Markov semigroup, Lindblad representation.

The paper is in final form and no version of it will be published elsewhere. 
bounded operators on a complex separable Hilbert space h, with a faithful normal invariant state $\rho$, was introduced by Alicki [3] (see also Frigerio, Gorini, Kossakowski, and Verri [12]): a QMS $\mathcal{T}$ generated by $\mathcal{L}$ satisfies the quantum detailed balance condition (QDB) if

$$
\operatorname{tr}(\rho x \mathcal{L}(y))-\operatorname{tr}(\rho \mathcal{L}(x) y)=2 i \operatorname{tr}(\rho[K, x] y),
$$

for some self-adjoint operator $K \in \mathcal{B}(\mathrm{h})$ and all $x, y \in \mathcal{B}(\mathrm{h})$.

This implies that the conditionally completely positive map $\widetilde{\mathcal{L}}$ defined by $\widetilde{\mathcal{L}}(x)=$ $\mathcal{L}(x)-2 i[K, x]$ coincides with the adjoint of $\mathcal{L}$ with respect to the pre-scalar product $\langle x, y\rangle_{0}:=\operatorname{tr}\left(\rho x^{*} y\right)$ on $\mathcal{B}(\mathrm{h})$. As a consequence, both $\mathcal{L}$ and $\widetilde{\mathcal{L}}$ commute with the modular group $\left(\sigma_{t}\right)_{t \in \mathbb{R}}$ associated with $\rho$ given by $\sigma_{t}(a)=\rho^{i t} a \rho^{-i t}$ ([12, Proposition 2.1), which is quite a restrictive condition (see e.g. [9], Theorems 3, 4 and 8, and also [14]). Moreover, the norm continuous semigroup $\widetilde{\mathcal{T}}=\left(\widetilde{\mathcal{T}}_{t}\right)_{t>0}$ generated by $\widetilde{\mathcal{L}}$, called the dual semigroup of $\mathcal{T}$, satisfies the corresponding equation $\operatorname{tr}\left(\rho x \mathcal{T}_{t}(y)\right)=\operatorname{tr}\left(\rho \widetilde{\mathcal{T}}_{t}(x) y\right)$ for all $t \geq 0$. The picture does not change if we consider pre-scalar products $\langle x, y\rangle_{s}:=\operatorname{tr}\left(\rho^{1-s} x^{*} \rho^{s} y\right)$ with $s \in[0,1], s \neq 1 / 2$, as shown in [7], Section 8 .

Complete positivity of the maps $\widetilde{\mathcal{T}}_{t}$ of the dual semigroup follows immediately without any additional condition if we consider the dual semigroup with respect to the above prescalar product on $\mathcal{B}(\mathrm{h})$ with $s=1 / 2$ as in 4, 11. The QDB condition obtained with the dual quantum Markov semigroup $\widetilde{\mathcal{T}}$ defined with respect to this scalar product is called standard detailed balance condition (SQDB) (see [5]).

Agarwal's original definition of quantum detailed balance [2], however, as well as its generalisations studied by Majewski [13, also keeps into account the parity of the observables $x, y$. This goal is achieved in physical applications by introducing an antiunitary time reversal $\theta$ and considering time-reversed observables $\theta x^{*} \theta$. It is reasonable to assume that $\theta$ commutes with $\rho$ because $\rho$ is typically a function of the energy of the system and thus it is invariant under time reversal (see [16]), i.e. $\theta \rho^{*} \theta=\theta \rho \theta=\rho$. The quantum detailed balance condition with time reversal $\theta(\mathrm{QDB}-\theta)$ is then defined by

$$
\operatorname{tr}\left(\rho x \mathcal{T}_{t}(y)\right)=\operatorname{tr}\left(\rho \theta y^{*} \theta \mathcal{T}_{t}\left(\theta x^{*} \theta\right)\right)
$$

for all $x, y \in \mathcal{B}(\mathrm{h})$ and $t \geq 0$. This, however, implies again commutation with the modular group that can be avoided by considering, as we proposed in [9, 10, the standard quantum detailed balance condition with time reversal $\theta$ (SQDB- $\theta)$ defined by

$$
\operatorname{tr}\left(\rho^{1 / 2} x \rho^{1 / 2} \mathcal{T}_{t}(y)\right)=\operatorname{tr}\left(\rho^{1 / 2} \theta y^{*} \theta \rho^{1 / 2} \mathcal{T}_{t}\left(\theta x^{*} \theta\right)\right)
$$

for all $x, y \in \mathcal{B}(\mathrm{h})$ and $t \geq 0$.

There are then essentially four types of quantum detailed balance conditions depending on the choice of the pre-scalar product with $s \neq 1 / 2$ or $s=1 / 2$ and on the inclusion or exclusion of an anti-unitary time reversal $\theta$ in the definition. In a series of papers (see e.g. [7, 9, 10]) we studied the structure of generators satisfying these four types of quantum detailed balance conditions. The results give several reasons to think that the best non-commutative version is obtained by considering the standard quantum detailed balance condition with an anti-unitary time reversal.

This paper brings another argument to support this conclusion. Indeed, we give a full classification and representation in a standard form of generators of quantum Markov 
semigroups on $\mathcal{B}(\mathrm{h})$, with $\mathrm{h} n$-dimensional, satisfying the standard quantum detailed balance condition with an anti-unitary time reversal, which generalises similar results for generators, commuting with the modular group, of QMSs satisfying the QDB- $\theta$ condition (see e.g. [9], Theorem 8).

The paper is organised as follows. In Section 2 we review our results on the structure of generators of QMSs satisfying one of the above quantum versions of the detailed balance condition. Standard forms of their generators, when the Hilbert space is finite-dimensional and the invariant state $\rho$ is generic (in a sense that will be clarified later), are given in Section 3 . Theorem 10 and Section 4 . Theorem 14.

As a consequence, we show that the convex cone of generators of QMSs satisfying the SQDB- $\theta$ (resp. QDB- $\theta$ ) condition, when looked at as a real manifold, has dimension growing as $n^{4} / 2$ (resp. $3 n^{2} / 2$ ).

We would like to stress here that understanding the quantum versions of the classical detailed balance conditions is the first step in the study of their generalisations for systems out of equilibrium like the Accardi-Imafuku dynamical detailed balance condition defined in [1].

2. Notation and preliminaries. Let $\mathrm{h}$ be a complex separable Hilbert space and consider a norm continuous quantum Markov semigroup (QMS) $\mathcal{T}=\left(\mathcal{T}_{t}\right)_{t \geq 0}$ on $\mathcal{B}(\mathrm{h})$ with a faithful normal invariant state $\rho$ with spectral representation $\sum_{j \geq 1} \rho_{j}\left|e_{j}\right\rangle\left\langle e_{j}\right|$ with respect to an orthonormal basis $\left(e_{j}\right)_{j \geq 1}$ of $\mathrm{h}$.

We recall (Parthasarathy [15], Theorem 30.10) that the generator $\mathcal{L}$ of $\mathcal{T}$ admits a Gorini-Kossakowski-Sudarshan-Lindblad (GKSL) representation with respect to $\rho$ of the form

$$
\mathcal{L}(x)=i[H, x]-\frac{1}{2} \sum_{k}\left(L_{k}^{*} L_{k} x-2 L_{k}^{*} x L_{k}+x L_{k}^{*} L_{k}\right)
$$

by means of operators $H=H^{*}$ and $\left(L_{k}\right)_{k \geq 1}$ in $\mathcal{B}(\mathrm{h})$ such that

(i) $\operatorname{tr}\left(\rho L_{k}\right)=0$ for each $k \geq 1$,

(ii) $\sum_{k \geq 1} L_{k}^{*} L_{k}$ is strongly convergent,

(iii) if $\sum_{k \geq 0}\left|c_{k}\right|^{2}<\infty$ and $c_{0}+\sum_{k \geq 1} c_{k} L_{k}=0$ for complex scalars $\left(c_{k}\right)_{k \geq 0}$ then $c_{k}=0$ for every $k \geq 0$.

A GKSL representation with the above properties will be called special.

Another family $\left\{H^{\prime}, L_{k}^{\prime}: k \geq 1\right\}$ of bounded operators in $\mathcal{B}(\mathrm{h})$ with $H^{\prime}$ self-adjoint satisfies equation (4) and conditions (i)-(iii) if and only if the lengths of the sequences $\left(L_{k}\right)_{k \geq 1},\left(L_{k}^{\prime}\right)_{k \geq 1}$ are equal and

$$
H^{\prime}=H+\alpha, \quad L_{k}^{\prime}=\sum_{j} u_{k j} L_{j}
$$

for some real scalar $\alpha$ and a unitary matrix $\left(u_{k j}\right)_{k j}$ on a Hilbert space k, called the multiplicity space of the completely positive part of $\mathcal{L}$.

Introducing the bounded operator

$$
G=-i H-\frac{1}{2} \sum_{k} L_{k}^{*} L_{k}
$$


on $\mathrm{h}$, we can also write $\mathcal{L}$ as

$$
\mathcal{L}(a)=G^{*} a+\sum_{k} L_{k}^{*} a L_{k}+a G .
$$

We call such a representation special if the operators $H=\left(G^{*}-G\right) / 2 i$ and $L_{k}$ give a special GKSL representation of $\mathcal{L}$. In this case, $G$ is unique up to a purely imaginary multiple of the identity operator. Indeed, if $G^{\prime},\left\{L_{k}^{\prime}\right\}$ give another special representation of $\mathcal{L}$, then $G^{\prime}=-i H^{\prime}-\frac{1}{2} \sum_{k} L_{k}^{\prime *} L_{k}^{\prime}$ fulfils

$$
G^{\prime}=-i H-i \alpha-\frac{1}{2} \sum_{k, j, m} \bar{u}_{k j} u_{k m} L_{j}^{*} L_{m}=G-i \alpha
$$

for some $\alpha \in \mathbb{R}$, since the matrix $\left(u_{k j}\right)_{k j}$ is unitary.

Following the terminology of [7, a special GKSL representation of $\mathcal{L}$ is called privileged if $H$ commutes with $\rho$ and the operators $L_{\ell}$ satisfy $\rho L_{\ell}=\lambda_{\ell} L_{\ell} \rho$ for some $\lambda_{\ell}>0$. In an equivalent way we can say that a special GKSL representation is privileged if its operator $H$ commutes with $\rho$ and the $L_{\ell}$ are eigenvectors of the modular group satisfying $\sigma_{t}\left(L_{\ell}\right)=\lambda_{\ell}^{i t} L_{\ell}$.

A special GKSL representation of the generator $\mathcal{L}$ of a norm-continuous QMS with respect to a given state $\rho$ always exists. This is not the case for privileged representations. Indeed, we have the following (see [7], Theorem 4.3).

THEOREM 1. The following conditions are equivalent:

(1) the generator $\mathcal{L}$ commutes with the modular group,

(2) there exists a privileged GKSL representation of $\mathcal{L}$,

(3) the dual semigroup $\widetilde{\mathcal{T}}$ with respect to pre-scalar products $\langle\cdot, \cdot\rangle_{s}$ with $s \neq 1 / 2$ is a $Q M S$.

We now list the characterisations of generators satisfying the QDB, SQDB, QDB- $\theta$ and SQDB- $\theta$ conditions referring to [7, 9, 10, for the proofs.

THEOREM 2. The QMS $\mathcal{T}$ satisfies the $Q D B$ condition (1) if and only if there exists a privileged GKSL representation of $\mathcal{L}$, by means of operators $H, L_{k}$, such that

(1) $H=K+c$ for some $c \in \mathbb{R}$,

(2) $\lambda_{\ell}^{-1 / 2} L_{\ell}^{*}=\sum_{j} u_{\ell j} L_{j}$ for some $\lambda_{\ell}>0$ and some $U=\left(u_{\ell j}\right)_{\ell j}$ unitary operator on $\mathrm{k}$ which is also symmetric (i.e. $u_{j k}=u_{k j}$ for all $j, k$ ).

It can be shown that all privileged GKSL representations enjoy the above properties. The proof can be found in [7], Theorem 5.1 and also [9], Theorem 14. The unitary $U$ is symmetric by the following algebraic argument. Noting that $\sigma_{i / 2}\left(L_{\ell}\right)=\lambda_{\ell}^{-1 / 2} L_{\ell}$, we can write $\lambda_{\ell}^{-1 / 2} L_{\ell}^{*}=\rho^{1 / 2} L_{\ell}^{*} \rho^{-1 / 2}$ and the identity $\lambda_{\ell}^{-1 / 2} L_{\ell}^{*}=\sum_{j} u_{\ell j} L_{j}$ becomes

$$
\rho^{1 / 2} L_{\ell}^{*}=\sum_{j} u_{\ell j} L_{j} \rho^{1 / 2} .
$$

Taking the adjoint we find $L_{\ell} \rho^{1 / 2}=\sum_{j} \bar{u}_{\ell j} \rho^{1 / 2} L_{j}^{*}$, i.e., by a change of indices, $L_{j} \rho^{1 / 2}=$ 
$\sum_{k} \bar{u}_{j k} \rho^{1 / 2} L_{k}^{*}$. Replacing this in the right-hand side of (6) we have

$$
\rho^{1 / 2} L_{\ell}^{*}=\sum_{j, k} u_{\ell j} \bar{u}_{j k} \rho^{1 / 2} L_{k}^{*}
$$

and the linear independence of the operators $\left(\rho^{1 / 2} L_{\ell}^{*}\right)_{\ell \geq 1}$ implies that $U \bar{U}$ is the identity operator, i.e. $\bar{U}=U^{*}$ because $U$ is unitary. This implies that $U$ coincides with its transpose.

THEOREM 3. The QMS $\mathcal{T}$ satisfies the $S Q D B$ condition

$$
\operatorname{tr}\left(\rho^{1 / 2} x \rho^{1 / 2} \mathcal{L}(y)\right)=\operatorname{tr}\left(\rho^{1 / 2} \widetilde{\mathcal{L}}(x) \rho^{1 / 2} y\right) \quad \text { and } \quad \mathcal{L}(x)-\widetilde{\mathcal{L}}(x)=2 i[K, x]
$$

for some self-adjoint operator $K$ if and only if there exists a special GKSL representation of the generator $\mathcal{L}$ by means of operators $G, L_{\ell}$ and there exists a unitary $\left(u_{j k}\right)_{j k}$ on $\mathrm{k}$ which is also symmetric (i.e. $u_{j k}=u_{k j}$ for all $j, k$ ) such that, for all $\ell \geq 1$, we have $\rho^{1 / 2} L_{\ell}^{*}=\sum_{k} u_{\ell k} L_{k} \rho^{1 / 2}$.

We refer to [10], Theorem 5 and Remark 4 for the proof (see also [9], Theorem 19).

We now consider quantum detailed balance conditions with an anti-unitary time reversal $\theta$. Recall that it satisfies $\theta \theta^{*}=\theta^{*} \theta=\mathbb{1}, \theta^{*}=\theta^{-1}$ and $\langle v, \theta u\rangle=\left\langle u, \theta^{*} v\right\rangle$ for all $u, v \in \mathrm{h}$.

THEOREM 4. The $Q M S \mathcal{T}$ satisfies the $Q D B-\theta$-condition (2) if and only if there exists a privileged GKSL representation of the generator $\mathcal{L}$, by means of operators $H$ and $L_{\ell}$ such that

(1) $H=\theta^{-1} H \theta+c$ for some $c \in \mathbb{R}$,

(2) $\lambda_{\ell}^{-1 / 2} \theta^{-1} L_{\ell}^{*} \theta=\sum_{j} u_{\ell j} L_{j}$ for some $\lambda_{\ell}>0$ and some $U=\left(u_{\ell j}\right)_{\ell j}$ unitary operator on $\mathrm{k}$ which is also self-adjoint.

The proof is given in 8], Theorem 9. Self-adjointness of $U$ follows from an argument similar to the above proof of symmetry (Theorem 2 recalling that $\theta$, as an anti-unitary operator, is anti-linear.

THEOREM 5. The QMS $\mathcal{T}$ satisfies the $S Q D B-\theta$ condition (3) if and only if there exists a special $G K S L$ representation of $\mathcal{L}$, with operators $G, L_{\ell}$, such that

(1) $\rho^{1 / 2} \theta^{-1} G^{*} \theta=G \rho^{1 / 2}$,

(2) $\rho^{1 / 2} \theta^{-1} L_{\ell}^{*} \theta=\sum_{j} u_{\ell j} L_{j} \rho^{1 / 2}$ for a self-adjoint unitary $\left(u_{\ell j}\right)_{\ell j}$ on $\mathrm{k}$.

We refer to [10], Theorem 8 for the proof.

Standard quantum detailed balance conditions do not imply commutation with the modular group, which is a purely algebraic constraint on the dynamics. However, when this algebraic condition holds, the SQDB and QDB, as well as the SQDB- $\theta$ and QDB- $\theta$ conditions, coincide (see e.g. 7], Theorem 7.1 and 4], Theorem 6.6).

There are two reasons convincing us that the anti-unitary time reversal $\theta$ must play some role. The first one is the physical motivation exposed in the introduction, namely keeping into account the parity of the observables ([2], [16]). The second one is the possibility to define, as discussed in [6], a natural notion of entropy production for QMS which is zero if and only if the SQDB- $\theta$ holds. Moreover, the following remark allows us to find a simple standard form for generators of QMSs satisfying the SQDB- $\theta$ condition. 
REMARK 6. Let $V=\left(v_{j k}\right)_{j k}$ be a unitary on the multiplicity space $\mathrm{k}$ of the completely positive part of $\mathcal{L}$ and define $L_{j}:=\sum_{j} v_{j k} M_{k}$. Then the identity $\rho^{1 / 2} \theta^{-1} L_{\ell}^{*} \theta=$ $\sum_{j} u_{\ell j} L_{j} \rho^{1 / 2}$ becomes $\rho^{1 / 2} \theta^{-1} M_{j}^{*} \theta=\sum_{k} w_{j k} M_{k} \rho^{1 / 2}$ where $W=\left(w_{j k}\right)_{j k}$ is the selfadjoint unitary $W=V^{*} U V$. In particular, since $U=\left(u_{\ell j}\right)_{\ell j}$ is unitary and self-adjoint, for a suitable choice of $V, W$ is a diagonal matrix with eigenvalues +1 and -1 and then either $\rho^{1 / 2} \theta^{-1} M_{j}^{*} \theta=M_{j} \rho^{1 / 2}$ or $\rho^{1 / 2} \theta^{-1} M_{j}^{*} \theta=-M_{j} \rho^{1 / 2}$ for all $j$.

Exploiting this simple remark, we shall describe explicitly the structure of generators $\mathcal{L}$ of QMS satisfying the SQDB- $\theta$ condition, generalising the analogous result for QMS satisfying the QDB and QDB- $\theta$ condition (see [9], Theorem 8 and Section 4 here).

Throughout the paper, for the sake of simplicity and concreteness, we shall consider a finite-dimensional Hilbert space $\mathbb{C}^{n}$ and QMS acting on the algebra $M_{n}(\mathbb{C})$ of $n \times$ $n$ complex matrices. $E_{k}^{j}$ denotes the rank one matrix $\left|e_{k}\right\rangle\left\langle e_{j}\right|$. We choose a canonical orthonormal basis $\left(e_{k}\right)_{k=1}^{n}$ where $\rho=\sum_{j=1}^{n} \rho_{j} E_{j}^{j}$ is diagonal. Clearly $0<\rho_{k}<1$ for all $k=1, \ldots, n$ since $\rho$ is faithful.

The anti-unitary time reversal $\theta$ will be just conjugation with respect to this basis, i.e. $\theta \sum_{j} z_{j} e_{j}=\sum_{j} \bar{z}_{j} e_{j}$. As a consequence $\theta^{-1} X^{*} \theta$ is the transpose $X^{T}$ and $\theta^{-1} X^{*} \theta=\bar{X}$, the matrix obtained by $X$ complex conjugating its entries.

3. SQDB- $\boldsymbol{\theta}$ condition. In this section, we find a standard form of a special GKSL representation of the generator $\mathcal{L}$ of a QMS $\mathcal{T}$ satisfying the SQDB- $\theta$. Since $\theta$ and $\rho$ commute, Theorem 5 says that this property holds if and only if the operators $G, L_{k}$ satisfy

(i) $G=\rho^{1 / 2} G^{T} \rho^{-1 / 2}$,

(ii) $L_{k}=\sum_{j \in \mathcal{J}} u_{k j} \rho^{1 / 2} L_{j}^{T} \rho^{-1 / 2}$ for some unitary self-adjoint $U=\left(u_{k j}\right)_{k, j \in \mathcal{J}}$.

In order to determine the structure of the operators $G$ and $L_{k}$ fulfilling (i) and (ii), we choose the basis $\left\{E_{l}^{l}, X_{i}^{j}, Y_{i}^{j}: l, i, j=1, \ldots, n, i<j\right\}$ of eigenvectors of the linear $\operatorname{map}$ on $M_{n}(\mathbb{C})$

$$
\mathcal{R}(X)=\rho^{1 / 2} X^{T} \rho^{-1 / 2},
$$

given by

$$
X_{h}^{j}:=\rho^{1 / 2}\left(E_{h}^{j}+E_{j}^{h}\right), \quad Y_{h}^{j}=i \rho^{1 / 2}\left(E_{j}^{h}-E_{h}^{j}\right)
$$

for $h<j$. In fact

$$
\mathcal{R}\left(E_{l}^{l}\right)=E_{l}^{l}, \quad \mathcal{R}\left(X_{h}^{j}\right)=X_{h}^{j}, \quad \mathcal{R}\left(Y_{h}^{j}\right)=-Y_{h}^{j}
$$

for all $l, h, j=1, \ldots, n, h<j$.

With respect to this basis we can write

$$
H=\sum_{l=1}^{n} h_{l} E_{l}^{l}+\sum_{1 \leq k<j \leq n}\left(m_{k j} X_{k}^{j}+w_{k j} Y_{k}^{j}\right)
$$

for some $h_{l}, m_{k j}, w_{k j} \in \mathbb{C}$. 
LEMma 7. The operator $H$ given by 10 is self-adjoint if and only if $h_{l} \in \mathbb{R}$ and

$$
\Im m_{k j}=\frac{\rho_{k}^{1 / 2}-\rho_{j}^{1 / 2}}{\rho_{k}^{1 / 2}+\rho_{j}^{1 / 2}} \Re w_{k j}, \quad \Im w_{k j}=\frac{\rho_{j}^{1 / 2}-\rho_{k}^{1 / 2}}{\rho_{k}^{1 / 2}+\rho_{j}^{1 / 2}} \Re m_{k j}
$$

for all $l, j, k=1, \ldots, n$ with $k<j$.

Proof. Set $Z_{k}^{j}:=m_{k j} X_{k}^{j}+w_{k j} Y_{k}^{j}=\rho_{k}^{1 / 2}\left(m_{k j}-i w_{k j}\right) E_{k}^{j}+\rho_{j}^{1 / 2}\left(m_{k j}+i w_{k j}\right) E_{j}^{k}$ for $k<j$. Since $H=\sum_{l=1}^{n} h_{l} E_{l}^{l}+\sum_{k<j} Z_{k}^{j}$ and

$$
\left(Z_{k}^{j}\right)^{*}=\rho_{k}^{1 / 2}\left(\bar{m}_{k j}+i \bar{w}_{k j}\right) E_{j}^{k}+\rho_{j}^{1 / 2}\left(\bar{m}_{k j}-i \bar{w}_{k j}\right) E_{k}^{j},
$$

$H$ is self-adjoint if and only if every $h_{l}$ is real and

$$
\left\{\begin{array}{l}
\rho_{k}^{1 / 2}\left(m_{k j}-i w_{k j}\right)=\rho_{j}^{1 / 2}\left(\bar{m}_{k j}-i \bar{w}_{k j}\right) \\
\rho_{j}^{1 / 2}\left(m_{k j}+i w_{k j}\right)=\rho_{k}^{1 / 2}\left(\bar{m}_{k j}+i \bar{w}_{k j}\right)
\end{array}\right.
$$

for all $k<j$. This system is easily solvable giving the equations (11) as unique solution.

Definition 8. Let $\left\{L_{k}\right\}_{k \in \mathcal{J}}$ be in a special representation of $\mathcal{L}$. We say that the operators $L_{k}$ are expressed in a standard form with respect to $\rho$ if $\mathcal{R}\left(L_{k}\right)=\epsilon_{k} L_{k}$ with $\epsilon_{k} \in\{-1,1\}$ for all $k \in \mathcal{J}$. In this case, the special representation of $\mathcal{L}$ is called standard.

Clearly every family of operators $\left\{L_{k}\right\}_{k \in \mathcal{J}}$ expressed in a standard form satisfies condition (ii) with $U=\operatorname{diag}\left(\epsilon_{1}, \ldots, \epsilon_{|\mathcal{J}|}\right)$, where $|\mathcal{J}|$ denotes the cardinality of $\mathcal{J}$. Conversely, if $L_{k}$ 's fulfil condition (ii) for some unitary self-adjoint matrix $U$, then we can suppose that they are expressed in a standard form. Indeed, by Remark 6, we have $U=V^{*} D V$ for some unitary matrix $V=\left(v_{i j}\right)_{i, j \in \mathcal{J}}$ and some diagonal matrix $D$ of the form

$$
\operatorname{diag}\left(\epsilon_{1}, \ldots, \epsilon_{|\mathcal{J}|}\right), \quad \epsilon_{i} \in\{-1,1\}
$$

Thus, replacing the $L_{k}$ 's by operators $L_{k}^{\prime}:=\sum_{j \in \mathcal{J}} v_{k j} L_{j}$ if necessary, we can take $U$ as in 12 .

REMARK 9. Let $\left\{L_{k}\right\}_{k \in \mathcal{J}}$ and $\left\{L_{k}^{\prime}\right\}_{k \in \mathcal{J}^{\prime}}$ be operators giving the same special standard representation of $\mathcal{L}$ such that

$$
L_{k}^{\prime}=\sum_{j \in \mathcal{J}} w_{k j} L_{j}
$$

for some unitary matrix $W=\left(w_{k j}\right)_{k \in \mathcal{J}^{\prime}, j \in \mathcal{J}}$. If $\mathcal{R}\left(L_{k}^{\prime}\right)=\epsilon_{k}^{\prime} L_{k}^{\prime}$ and $\mathcal{R}\left(L_{j}\right)=\epsilon_{j} L_{j}$ with $\epsilon_{j}, \epsilon_{k}^{\prime} \in\{-1,1\}$ for all $k, j$, then

$$
w_{k j}\left(\epsilon_{k}^{\prime}-\epsilon_{j}\right)=0 \quad \text { for all } j \in \mathcal{J}, k \in \mathcal{J}^{\prime} .
$$

Indeed, applying the operator $\mathcal{R}$ to both sides of equation 13 , we get

$$
\epsilon_{k}^{\prime} \sum_{j \in \mathcal{J}} w_{k j} L_{j}=\epsilon_{k}^{\prime} L_{k}^{\prime}=\sum_{j \in \mathcal{J}} w_{k j} \epsilon_{j} L_{j}
$$

which clearly gives condition (14) by the linear independence of $L_{j}$ 's.

We can now characterise the Hamiltonian $H$ and the standard form of operators $L_{k}$ in a special GKSL representation of the generator under the SQDB- $\theta$. 
THEOREM 10. The $Q M S \mathcal{T}$ satisfies the $S Q D B-\theta$ with respect to $\rho$ if and only if there exists a special standard GKSL representation of $\mathcal{L}$ given by operators

$$
\begin{array}{rlrl}
H & =\sum_{l=1}^{n} h_{l} E_{l}^{l}+\sum_{h<j}\left(m_{h j} X_{h}^{j}+w_{h j} Y_{h}^{j}\right) & \\
L_{k} & =\sum_{l=1}^{n} v_{l}^{(k)} E_{l}^{l}+\sum_{h<j} \xi_{h j}^{(k)} X_{h}^{j} \quad \text { for } k \in \mathcal{J}^{+} \subseteq\left\{1, \ldots, \frac{n(n+1)}{2}-1\right\} \\
L_{k} & =\sum_{h<j} \zeta_{h j}^{(k)} Y_{h}^{j} & \text { for } k \in \mathcal{J}^{-} \subseteq\left\{\frac{n(n+1)}{2}, \ldots, n^{2}-1\right\},
\end{array}
$$

with

$$
\begin{aligned}
& \left\{\left(v_{1}^{(k)}, \ldots, v_{n-1}^{(k)}, \xi_{12}^{(k)}, \ldots, \xi_{1 n}^{(k)}, \ldots, \xi_{n-2 n-1}^{(k)}, \xi_{n-2 n}^{(k)}, \xi_{n-1 n}^{(k)}\right): k \in \mathcal{J}^{+}\right\} \\
& \left\{\left(\zeta_{12}^{(k)}, \ldots, \zeta_{1 n}^{(k)}, \ldots, \zeta_{n-2 n-1}^{(k)}, \zeta_{n-2 n}^{(k)}, \zeta_{n-1 n}^{(k)}\right): k \in \mathcal{J}^{-}\right\}
\end{aligned}
$$

sets of linearly independent vectors in $\mathbb{C}^{n(n+1) / 2-1}$ and $\mathbb{C}^{n(n-1) / 2}$ respectively,

$$
v_{n}^{(k)}=-\sum_{l=1}^{n-1} v_{l}^{(k)} \rho_{l} \rho_{n}^{-1} \quad \text { for } k \in \mathcal{J}^{+},
$$

$h_{l} \in \mathbb{R}, m_{h j}$ given by f11 for all $h<j$, and

$$
w_{h j}=\frac{1}{2} \operatorname{tr}\left(\rho_{h}^{-1 / 2}\left(G_{0}\right)_{h j}-\rho_{j}^{-1 / 2}\left(G_{0}\right)_{j h}\right)=\frac{i}{2} \operatorname{tr}\left(Y_{h}^{j} \rho^{-1 / 2} G_{0} \rho^{-1 / 2}\right),
$$

where $G_{0}:=-2^{-1} \sum_{k \in \mathcal{J}^{+} \cup \mathcal{J}^{-}} L_{k}^{*} L_{k}$.

Proof. Assume that SQDB- $\theta$ holds and let $\left\{L_{k}, H: k \in \mathcal{J}\right\}$ be in a special GKSL representation of $\mathcal{L}$, with $L_{k}$ 's expressed in a standard form. The Hamiltonian $H$ is clearly given by 15 with $h_{l} \in \mathbb{R}$ and $m_{h j}, w_{h j}$ satisfying equations (11) thanks to Lemma 7 .

Since condition (ii) gives either $L_{k}=\rho^{1 / 2} L_{k}^{T} \rho^{-1 / 2}=\mathcal{R}\left(L_{k}\right)$ or $L_{k}=-\rho^{1 / 2} L_{k}^{T} \rho^{-1 / 2}=$ $-\mathcal{R}\left(L_{k}\right)$, expanding $L_{k}$ on the basis $\left\{E_{l}^{l}, X_{i}^{j}, Y_{i}^{j}: l, i, j=1, \ldots, n, i<j\right\}$ and recalling equation (9), we get

$$
\begin{aligned}
L_{k}=\rho^{1 / 2} L_{k}^{T} \rho^{-1 / 2} & \Leftrightarrow L_{k}=\sum_{l=1}^{n} v_{l}^{(k)} E_{l}^{l}+\sum_{h<j} \xi_{h j}^{(k)} X_{h}^{j}, \\
L_{k}=-\rho^{1 / 2} L_{k}^{T} \rho^{-1 / 2} & \Leftrightarrow L_{k}=\sum_{h<j} \zeta_{h j}^{(k)} Y_{h}^{j}
\end{aligned}
$$

for some $v_{l}^{(k)}, \xi_{h j}^{(k)}, \zeta_{h j}^{(k)} \in \mathbb{C}$, with $l, h, j=1, \ldots, n$ and $h<j$. Assume that 20 and (21) hold for $k \in \mathcal{J}^{+}$and $k \in \mathcal{J}^{-}$, respectively, with $\mathcal{J}^{+}$and $\mathcal{J}^{-}$disjoint subsets of $\mathcal{J}$ such that $\mathcal{J}^{+} \cup \mathcal{J}^{-}=\mathcal{J}$. Therefore, condition $\operatorname{tr}\left(\rho L_{k}\right)=0$ is clearly always satisfied if $k \in \mathcal{J}^{-}$, while it means

$$
\sum_{l=1}^{n} \rho_{l} v_{l}^{(k)}=0, \quad \text { i.e. } \quad v_{n}^{(k)}=-\sum_{l=1}^{n-1} v_{l}^{(k)} \rho_{l} \rho_{n}^{-1}
$$

for each $k \in \mathcal{J}^{+}$. Finally, by the linear independence of $\left\{\mathbb{1}, L_{k}: k \in \mathcal{J}\right\}$, the inequalities

$$
|\mathcal{J}| \leq n^{2}-1, \quad\left|\mathcal{J}^{+}\right| \leq n-1+n(n-1) / 2=n(n+1) / 2-1, \quad\left|\mathcal{J}^{-}\right| \leq n(n-1) / 2,
$$


hold and the vectors in $\mathbb{C}^{n(n+1) / 2-1}$ and $\mathbb{C}^{n(n-1) / 2}$ respectively

$$
\begin{aligned}
& \left\{\left(v_{1}^{(k)}, \ldots, v_{n-1}^{(k)}, \xi_{12}^{(k)}, \ldots, \xi_{1 n}^{(k)}, \ldots, \xi_{n-2 n-1}^{(k)}, \xi_{n-2 n}^{(k)}, \xi_{n-1 n}^{(k)}\right): k \in \mathcal{J}^{+}\right\} \\
& \left\{\left(\zeta_{12}^{(k)}, \ldots, \zeta_{1 n}^{(k)}, \ldots, \zeta_{n-2 n-1}^{(k)}, \zeta_{n-2 n}^{(k)}, \zeta_{n-1 n}^{(k)}\right): k \in \mathcal{J}^{-}\right\}
\end{aligned}
$$

must be linearly independent.

Now, since

$$
G=-i H-2^{-1} \sum_{k} L_{k}^{*} L_{k}=-i\left[\sum_{l=1}^{n} h_{l} E_{l}^{l}+\sum_{h<j}\left(m_{h j} X_{h}^{j}+w_{h j} Y_{h}^{j}\right)\right]+G_{0},
$$

in view of $(9)$, condition (i), i.e. $G=\mathcal{R}(G)$, reads $-2 i\left(\sum_{h<j} w_{h j} Y_{h}^{j}\right)+G_{0}=\mathcal{R}\left(G_{0}\right)$, that is

$$
2 i \sum_{h<j} w_{h j} Y_{h}^{j}=G_{0}-\rho^{1 / 2} G_{0}^{T} \rho^{-1 / 2}
$$

Note that

$$
\operatorname{tr}\left(\left(E_{l}^{m}-E_{m}^{l}\right)\left(E_{j}^{h}-E_{h}^{j}\right)\right)=2 \delta_{m j} \delta_{l h}-2 \delta_{m h} \delta_{l j}
$$

and so, left multiplying both sides of equation 22 by $\left(E_{l}^{m}-E_{m}^{l}\right) \rho^{-1 / 2}$ with $m<l$ and tracing, we get

$$
-4 i w_{m l}=\operatorname{tr}\left(\left(E_{l}^{m}-E_{m}^{l}\right)\left(\rho^{-1 / 2} G_{0}-G_{0}^{T} \rho^{-1 / 2}\right)\right) .
$$

Now, since $\operatorname{tr}\left(E_{l}^{m} \rho^{-1 / 2} G_{0}\right)=\operatorname{tr}\left(G_{0}^{T} \rho^{-1 / 2} E_{m}^{l}\right)=\operatorname{tr}\left(E_{m}^{l} G_{0}^{T} \rho^{-1 / 2}\right)$, we can write the previous relation as

$$
\begin{aligned}
w_{m l}=\frac{i}{2} \operatorname{tr}\left(\left(E_{l}^{m}-E_{m}^{l}\right) \rho^{-1 / 2} G_{0}\right)=\frac{1}{2} \operatorname{tr}\left(Y_{m}^{l} \rho^{-1 / 2} G_{0} \rho^{-1 / 2}\right) & \\
& =\frac{i}{2}\left(\rho_{m}^{-1 / 2}\left(G_{0}\right)_{m l}-\rho_{l}^{-1 / 2}\left(G_{0}\right)_{l m}\right) .
\end{aligned}
$$

COROLlaRY 11. If $\mathcal{T}$ satisfies the $S Q D B-\theta$, then every special standard representation of $\mathcal{L}$ is of the form given in Theorem 10 .

Proof. Let $\left\{H, L_{k}, L_{l}: k \in \mathcal{J}^{+}, l \in \mathcal{J}^{-}\right\}$be a special standard representation of $\mathcal{L}$ defined as in 15-17) with $h_{l} \in \mathbb{R}, m_{h j}, w_{h j}$ given for all $h<j$ by 11] and 19 respectively,

$$
\begin{aligned}
& \left\{\left(v_{1}^{(k)}, \ldots, v_{n-1}^{(k)}, \xi_{12}^{(k)}, \ldots, \xi_{1 n}^{(k)}, \ldots, \xi_{n-2 n-1}^{(k)}, \xi_{n-2 n}^{(k)}, \xi_{n-1 n}^{(k)}\right): k \in \mathcal{J}^{+}\right\} \\
& \left\{\left(\zeta_{12}^{(k)}, \ldots, \zeta_{1 n}^{(k)}, \ldots, \zeta_{n-2 n-1}^{(k)}, \zeta_{n-2 n}^{(k)}, \zeta_{n-1 n}^{(k)}\right): k \in \mathcal{J}^{-}\right\}
\end{aligned}
$$

sets of linearly independent vectors in $\mathbb{C}^{n(n+1) / 2-1}$ and $\mathbb{C}^{n(n-1) / 2}$ respectively and let $v_{n}^{(k)}$ for $k \in \mathcal{J}^{+}$be as in 18 .

If we consider $\left\{\widetilde{H}, \widetilde{L}_{k}\right\}_{k \in \widetilde{\mathcal{J}}}$ another special standard representation of $\mathcal{L}$, then we have $\left|\mathcal{J}^{+}\right|+\left|\mathcal{J}^{-}\right|=|\widetilde{\mathcal{J}}|$ and

$$
\widetilde{L}_{k}=\sum_{j \in \mathcal{J}^{+}} u_{k j} L_{j}+\sum_{j \in \mathcal{J}^{-}} u_{k j} L_{j}, \quad \widetilde{H}=H+\alpha
$$

for some unitary matrix $U=\left(u_{k j}\right)_{k \in \mathcal{J}, j \in \mathcal{J}}$ and real constant $\alpha$. Since $\mathcal{R}\left(\widetilde{L}_{k}\right)=\widetilde{\epsilon}_{k} \widetilde{L}_{k}$ with $\widetilde{\epsilon}_{k} \in\{-1,1\}$, and

$$
\mathcal{R}\left(L_{j}\right)= \begin{cases}L_{j} & \text { if } j \in \mathcal{J}^{+} \\ -L_{j} & \text { if } j \in \mathcal{J}^{-}\end{cases}
$$


defining $\widetilde{\mathcal{J}}^{+}:=\left\{k \in \tilde{\mathcal{J}}: \widetilde{\epsilon}_{k}=1\right\}$ and $\widetilde{\mathcal{J}}^{-}:=\left\{k \in \tilde{\mathcal{J}}: \widetilde{\epsilon}_{k}=-1\right\}$ we get $u_{k j}=0$ for all $(k, j) \in\left(\widetilde{\mathcal{J}}^{+} \times \widetilde{\mathcal{J}}^{-}\right) \cup\left(\tilde{\mathcal{J}}^{-} \times \widetilde{\mathcal{J}}^{+}\right)$thanks to Remark 9 . This means that

$$
\widetilde{L}_{k}= \begin{cases}\sum_{j \in \mathcal{J}^{+}} u_{k j} L_{j} & \text { if } k \in \tilde{\mathcal{J}}^{+} \\ \sum_{j \in \mathcal{J}^{-}} u_{k j} L_{j} & \text { if } k \in \tilde{\mathcal{J}}^{-}\end{cases}
$$

i.e.

$$
\begin{array}{ll}
\widetilde{L}_{k}=\sum_{l=1}^{n}\left(\sum_{j \in \mathcal{J}^{+}} u_{k j} v_{l}^{(j)}\right) E_{l}^{l}+\sum_{h<l}\left(\sum_{j \in \mathcal{J}^{+}} u_{k j} \xi_{h l}^{(j)}\right) X_{h}^{j} & \text { if } k \in \widetilde{\mathcal{J}}^{+}, \\
\widetilde{L}_{k}=\sum_{h<l}\left(\sum_{j \in \mathcal{J}^{+}} u_{k j} \zeta_{h l}^{(j)}\right) Y_{h}^{l} & \text { if } k \in \widetilde{\mathcal{J}}^{-},
\end{array}
$$

with $\widetilde{\mathcal{J}}^{+} \subseteq\left\{1, \ldots, \frac{n(n+1)}{2}-1\right\}$ and $\widetilde{\mathcal{J}}^{-} \subseteq\left\{\frac{n(n+1)}{2}, \ldots, n^{2}-1\right\}$ by linear independence of $\widetilde{L}_{k}$. Set now

$$
\begin{gathered}
\widetilde{v}_{l}^{(k)}:=\sum_{j \in \mathcal{J}^{+}} u_{k j} v_{l}^{(j)}, \quad \widetilde{\xi}_{h l}^{(k)}:=\sum_{j \in \mathcal{J}^{+}} u_{k j} \xi_{h l}^{(j)} \quad \text { for } k \in \widetilde{\mathcal{J}}^{+} \\
\widetilde{\zeta}_{h l}^{(k)}:=\sum_{j \in \mathcal{J}^{+}} u_{k j} \zeta_{h l}^{(j)} \quad \text { for } k \in \widetilde{\mathcal{J}}^{-}
\end{gathered}
$$

Therefore, by equation (18) we have

$$
\widetilde{v}_{l}^{(k)}=-\sum_{j \in \mathcal{J}^{+}} u_{k j} \sum_{l=1}^{n-1} v_{l}^{(j)} \rho_{l} \rho_{n}^{-1}=-\sum_{l=1}^{n-1} \widetilde{v}_{l}^{(k)} \rho_{l} \rho_{n}^{-1} .
$$

Moreover, by the invertibility of $U$,

$$
\begin{aligned}
& \left\{\left(\widetilde{v}_{1}^{(k)}, \ldots, \widetilde{v}_{n-1}^{(k)}, \widetilde{\xi}_{12}^{(k)}, \ldots, \widetilde{\xi}_{1 n}^{(k)}, \ldots, \widetilde{\xi}_{n-2 n-1}^{(k)}, \widetilde{\xi}_{n-2 n}^{(k)}, \widetilde{\xi}_{n-1 n}^{(k)}\right): k \in \widetilde{\mathcal{J}}^{+}\right\} \\
& \left\{\left(\widetilde{\zeta}_{12}^{(k)}, \ldots, \widetilde{\zeta}_{1 n}^{(k)}, \ldots, \widetilde{\zeta}_{n-2 n-1}^{(k)}, \widetilde{\zeta}_{n-2 n}^{(k)}, \widetilde{\zeta}_{n-1 n}^{(k)}\right): k \in \widetilde{\mathcal{J}}^{-}\right\}
\end{aligned}
$$

are sets of linearly independent vectors in $\mathbb{C}^{n(n+1) / 2-1}$ and $\mathbb{C}^{n(n-1) / 2}$, respectively.

Finally, since $\widetilde{H}=H+\alpha$, it clearly has the form of equation 15 with real coefficients $\widetilde{h}_{l}=h_{l}+\alpha$ for all $l=1, \ldots, n$ and $\widetilde{m}_{h j}=m_{h j}, \widetilde{w}_{h j}=w_{h j}$ for all $h<j$.

REMARK 12. Theorem 10 shows that, in the generic case when the $\rho_{i}$ 's are all different, generators of QMSs with a faithful invariant state $\rho$ satisfying the SQDB- $\theta$ condition are parametrised by

(a) a set of $\frac{n(n+1)}{2}-1=\frac{(n-1)(n+2)}{2}$ linearly independent vectors in $\mathbb{C}^{(n-1)(n+1) / 2}$,

(b) a set of $n(n-1) / 2$ linearly independent vectors in $\mathbb{C}^{n(n-1) / 2}$,

(c) $n-1$ real parameters $h_{l}$ for the Hamiltonian $H$, since one of the $h_{l}$ can be eliminated by adding a constant multiple of the identity operator without changing $\mathcal{L}$, constants $m_{h j}$ are completely determined by $w_{h j}$ through (11) (for the eigenvalues of $\rho$ are all different) and any $w_{h j}$ is given by $G_{0}$ through (19).

Two sets of linearly independent vectors $u=\left(u_{1}, \ldots, u_{d}\right), u^{\prime}=\left(u_{1}^{\prime}, \ldots, u_{d}^{\prime}\right)$ in $\mathbb{C}^{d}$ (with $d=(n-1)(n+2) / 2$ or $d=n(n-1) / 2)$ lead to the same generator if there exists a unitary operator $U$ on $\mathbb{C}^{d}$ such that $U u=u^{\prime}$. Therefore the $L_{k}$ 's with $k \in \mathcal{J}^{+}$(resp. 
$k \in \mathcal{J}^{-}$) are parametrized by the linear manifold $G L(d, \mathbb{C}) / U(d)$ of invertible matrices modulo unitary matrices with $d=(n-1)(n+2) / 2(\operatorname{resp} . d=n(n-1) / 2)$.

The real dimension $G L(d, \mathbb{C})$ is clearly $2 d^{2}$. Moreover, norm preservation of $d$ vectors and scalar products preservation of pairs of $d(d-1)$ pairs of vectors give $d$ and $d(d-1)$ respectively identities involving the real and imaginary parts of these vectors. Thus we remain with $2 d^{2}-d(d-1)-d=d^{2}$ free real parameters.

It follows then that the convex cone of generators of QMSs satisfying the SQDB- $\theta$ condition, when the invariant state $\rho$ is non-degenerate, is a manifold with real dimension

$$
\left(\frac{(n+2)(n-1)}{2}\right)^{2}+\left(\frac{n(n-1)}{2}\right)^{2}+(n-1)=\frac{n^{2}\left(n^{2}-1\right)}{2} .
$$

4. QDB- $\theta$ condition in the generic case. In this section we analyse the QDB- $\theta$ condition for $\mathcal{T}$ under the hypothesis that the faithful invariant density $\rho$ is generic, i.e. its eigenvalues are all different and so are their ratios $\rho_{j} / \rho_{k}$ for $j \neq k$. The time reversal $\theta$ is still the usual conjugation on $\mathbb{C}^{n}$.

We recall that, by Theorem 4, the QDB- $\theta$ condition holds if and only if there exists a special GKSL representation of $\mathcal{L}$, by operators $H, L_{k}$ such that

(i) $[H, \rho]=0$,

(ii) $\rho L_{k}=\lambda_{k} L_{k} \rho$ for some $\lambda_{k} \in \mathbb{R}$,

(iii) $H=\bar{H}$,

(iv) $\lambda_{k}^{-1 / 2} L_{k}=\sum_{j} u_{k j} L_{j}^{T}$ for some $\lambda_{k}>0$ and some unitary self-adjoint matrix $U=$ $\left(u_{k j}\right)_{k, j \in \mathcal{J}}$.

A special representation satisfying (i) and (ii) is called privileged; moreover, see e.g. [7, the existence of a privileged representation of $\mathcal{L}$ is equivalent to the commutation of every $\mathcal{T}_{t}$ with the modular automorphism $\sigma_{-i}$ defined by $\rho, \sigma_{-i}(x)=\rho x \rho^{-1}$ for $x \in M_{n}(\mathbb{C})$. In particular, condition (ii) means that each $L_{k}$ is an eigenvector of the modular automorphism with eigenvalue $\lambda_{k}=\rho_{j} \rho_{l}^{-1}$ for each $j, l \in\{1, \ldots, n\}$ for which $\left\langle e_{j}, L_{k} e_{l}\right\rangle \neq 0$; it follows that, in the generic case, there can exist a unique pair $(j, l)$ with $j \neq l$ such that $\left\langle e_{j}, L_{k} e_{l}\right\rangle \neq 0$.

This remark allows us to characterise privileged GKSL representations of $\mathcal{L}$.

Proposition 13. Every privileged GKSL representation of $\mathcal{L}$ with respect to the generic and faithful state $\rho$ is of the form

$$
\begin{aligned}
\mathcal{L}(a) & =i[H, a]-\frac{1}{2} \sum_{k \in \mathcal{J}^{+}}\left(L_{k}^{*} L_{k} a-2 L_{k}^{*} a L_{k}+a L_{k}^{*} L_{k}\right) \\
& -\frac{1}{2} \sum_{j<l} \gamma_{j l}^{+}\left(E_{j}^{j} a-2 E_{j}^{l} a E_{l}^{j}+a E_{j}^{j}\right)-\frac{1}{2} \sum_{j<l} \gamma_{l j}^{-}\left(E_{l}^{l} a-2 E_{l}^{j} a E_{j}^{l}+a E_{l}^{l}\right),
\end{aligned}
$$

with $\gamma_{j l}^{+}, \gamma_{l j}^{-} \geq 0$ for all $j<l$,

$$
\begin{aligned}
H & =\sum_{i=1}^{n} h_{i} E_{i}^{i}, \quad h_{i} \in \mathbb{R} \quad \text { for all } i=1, \ldots, n, \\
L_{k} & =\sum_{j=1}^{n} z_{k j} E_{j}^{j} \quad \text { for } k \in \mathcal{J}_{1} \subseteq\{1, \ldots, n-1\},
\end{aligned}
$$


where

$$
z_{k n}=-\sum_{i=1}^{n-1} z_{k i} \rho_{i} \rho_{n}^{-1}
$$

and $\left\{z_{k}: k \in \mathcal{J}_{1}\right\}\left(z_{k}:=\left(z_{k 1}, \ldots, z_{k n-1}\right)\right)$ is a set of linearly independent vectors in $\mathbb{C}^{n-1}$. Moreover, the state $\rho$ is invariant if and only if the constants $\gamma_{j l}^{ \pm}$satisfy

$$
\left\{\begin{array}{l}
\sum_{l=2}^{n}\left(\rho_{l} \gamma_{l 1}^{-}-\rho_{1} \gamma_{1 l}^{+}\right)=0 \\
\sum_{j=1}^{i-1}\left(\rho_{i} \gamma_{i j}^{-}-\rho_{j} \gamma_{j i}^{+}\right)=\sum_{l=i+1}^{n}\left(\rho_{l} \gamma_{l i}^{-}-\rho_{i} \gamma_{i l}^{+}\right)
\end{array}\right.
$$

for $i=2, \ldots, n-1$.

Proof. Let $\left\{H, L_{k}: k \in \mathcal{J}\right\}$ be operators of a special GKSL representation of $\mathcal{L}$; the linear independence of $\left\{\mathbb{1}, L_{k}: k \in \mathcal{J}\right\}$ immediately gives $\operatorname{card}(\mathcal{J}) \leq n^{2}-1$. Moreover, if we write $H$ with respect to the basis $\left\{E_{l}^{j}\right\}_{l, j=1}^{n}$, its commutation with $\rho$ is clearly equivalent to having $H=\sum_{j=1}^{n} h_{j} E_{j}^{j}$, with $h_{j} \in \mathbb{R}$ for $H=H^{*}$.

We fix now $k \in \mathcal{J}$ and suppose $\rho L_{k}=\lambda_{k} L_{k} \rho$ for some $\lambda_{k} \in \mathbb{R}$; this means

$$
\rho_{j}\left\langle e_{j}, L_{k} e_{l}\right\rangle=\lambda_{k} \rho_{l}\left\langle e_{j}, L_{k} e_{l}\right\rangle \quad \text { for all } j, l=1, \ldots, n \text {. }
$$

Since $L_{k} \neq 0$, there exists at least a pair $(j, l)$ such that $\left\langle e_{j}, L_{k} e_{l}\right\rangle \neq 0$, and so $\lambda_{k}=\rho_{j} \rho_{l}^{-1}$. If $j=l$ (i.e. $\lambda_{k}=1$ ), $L_{k}$ must be diagonal with respect to the basis $\left\{E_{i}^{j}\right\}_{i, j}$, since the eigenvalues of $\rho$ are all distinct; therefore, we get

$$
L_{k}=\sum_{j=1}^{n} z_{k j} E_{j}^{j}, \quad z_{k j} \in \mathbb{C},
$$

with $0=\operatorname{tr}\left(\rho L_{k}\right)=\sum_{j=1}^{n} \rho_{j} z_{k j}$, namely

$$
z_{k n}=-\sum_{j=1}^{n-1} \frac{\rho_{j}}{\rho_{n}} z_{k j} .
$$

We denote by $\mathcal{J}_{1}$ the set of indexes $k \in \mathcal{J}$ such that the corresponding $L_{k}$ has the form 24); the linear independence condition gives then $\operatorname{card}\left(\mathcal{J}_{1}\right) \leq n-1$ with $\left\{z_{k}: k \in \mathcal{J}_{1}\right\}$ linearly independent vectors in $\mathbb{C}^{n-1}$, where we defined $z_{k}:=\left(z_{k 1}, \ldots, z_{k n-1}\right)$.

If, otherwise, $j \neq l$, as already mentioned before, the assumption on $\rho_{i}$ 's forces to have a unique pair $(j, l)$ satisfying $\lambda_{k}=\rho_{j} \rho_{l}^{-1}$, so that $L_{k}$ is a multiple of $E_{j}^{l}$. We set

$$
L_{k}= \begin{cases}\alpha_{l j}^{+} E_{j}^{l}=: L_{l j}^{+} & \text {if } j>l \\ \alpha_{j l}^{-} E_{j}^{l}=: L_{j l}^{-} & \text {if } j<l,\end{cases}
$$

with $\alpha_{j l}^{ \pm} \in \mathbb{C}$. Note that the operators $L_{j l}^{+}, L_{j l}^{-}$are linearly independent for all $j<l$, and they satisfy $\operatorname{tr}\left(\rho L_{j l}^{+}\right)=0=\operatorname{tr}\left(\rho L_{j l}^{-}\right)$. Moreover, up to a suitable choice of the phase factor for each $L_{j l}^{ \pm}$, we can suppose $\alpha_{j l}^{ \pm} \in \mathbb{R}$. We define

$$
\gamma_{j l}^{+}:=\left(\alpha_{j l}^{+}\right)^{2}, \quad \gamma_{l j}^{-}:=\left(\alpha_{j l}^{-}\right)^{2} \quad \text { for all } j<l .
$$


Conversely, it can easily be shown that the representation given by the operators $H=\sum_{j=1}^{n} h_{j} E_{j}^{j}$ with $h_{j} \in \mathbb{R}, L_{k}$ and $L_{j l}^{ \pm}$defined as in 24, 25) respectively, with $z_{k n}=-\sum_{j=1}^{n-1} \rho_{j} \rho_{n}^{-1} z_{k j}, \alpha_{j l}^{ \pm} \in \mathbb{R}$ and $\gamma_{j l}^{ \pm}$satisfying (26), is a privileged representation of $\mathcal{L}$ with respect to $\rho$.

Finally, we analyse the condition of the invariance of $\rho$. Since $\rho$ is faithful and the equalities $[H, \rho]=0, \rho L_{k}^{*} L_{k}=L_{k}^{*} L_{k} \rho, L_{k} \rho L_{k}^{*}=\lambda_{k}^{-1} L_{k} L_{k}^{*} \rho$ hold for every privileged representation, it follows that $\rho$ is an invariant state if and only if

$$
\sum_{k \in \mathcal{J}}\left(L_{k}^{*} L_{k}-\lambda_{k}^{-1} L_{k} L_{k}^{*}\right)=0 .
$$

Since $\lambda_{k}=1$ for all $k \in \mathcal{J}_{1}$, this gives

$$
\begin{aligned}
0 & =\sum_{j<l}\left(\left(L_{j l}^{+}\right)^{*} L_{j l}^{+}-\frac{\rho_{j}}{\rho_{l}} L_{j l}^{+}\left(L_{j l}^{+}\right)^{*}+\left(L_{j l}^{-}\right)^{*} L_{j l}^{-}-\frac{\rho_{l}}{\rho_{j}} L_{j l}^{-}\left(L_{j l}^{-}\right)^{*}\right) \\
& =\sum_{j<l}\left\{\gamma_{j l}^{+}\left(E_{j}^{j}-\frac{\rho_{j}}{\rho_{l}} E_{l}^{l}\right)+\gamma_{l j}^{-}\left(E_{l}^{l}-\frac{\rho_{l}}{\rho_{j}} E_{j}^{j}\right)\right\} \\
& =\sum_{j<l}\left\{\left(\gamma_{j l}^{+}-\frac{\rho_{l}}{\rho_{j}} \gamma_{l j}^{-}\right) E_{j}^{j}+\left(\gamma_{l j}^{-}-\frac{\rho_{j}}{\rho_{l}} \gamma_{j l}^{+}\right) E_{l}^{l}\right\},
\end{aligned}
$$

i.e.

$$
\left\{\begin{array}{l}
\sum_{l=2}^{n}\left(\gamma_{1 l}^{+}-\frac{\rho_{l}}{\rho_{1}} \gamma_{l 1}^{-}\right)=0 \\
\sum_{l=i+1}^{n}\left(\gamma_{i l}^{+}-\frac{\rho_{l}}{\rho_{i}} \gamma_{l i}^{-}\right)+\sum_{j=1}^{i-1}\left(\gamma_{i j}^{-}-\frac{\rho_{j}}{\rho_{i}} \gamma_{j i}^{+}\right)=0 \quad \text { for } i=2, \ldots, n-1 \\
\sum_{j=1}^{n-1}\left(\gamma_{n j}^{-}-\frac{\rho_{j}}{\rho_{n}} \gamma_{j n}^{+}\right)=0 .
\end{array}\right.
$$

Denoting by $R_{i}$ for $i=1, \ldots, n$ the left-hand side of the $i$-th equation in the above system, we can easily see that $R_{n}=-\rho_{1} \rho_{n}^{-1}\left(R_{1}+\sum_{i=2}^{n-1} R_{i}\right)$, and so the last equation in (27) is superfluous. Therefore, the condition of invariance can be read as

$$
\left\{\begin{array}{l}
\sum_{l=2}^{n}\left(\gamma_{1 l}^{+}-\frac{\rho_{l}}{\rho_{1}} \gamma_{l 1}^{-}\right)=0 \\
\sum_{j=1}^{i-1}\left(\gamma_{i j}^{-}-\frac{\rho_{j}}{\rho_{i}} \gamma_{j i}^{+}\right)=\sum_{l=i+1}^{n}\left(\frac{\rho_{l}}{\rho_{i}} \gamma_{l i}^{-}-\gamma_{i l}^{+}\right)
\end{array}\right.
$$

for $i=2, \ldots, n-1$.

We can now characterise generators of QMSs satisfying the QDB- $\theta$. Recall that, by Theorem 4 this condition is fulfilled if and only if there exists a privileged GKSL representation of $\mathcal{L}$, by means of operators $H$ and $L_{k}$, such that: (1) $H=\bar{H},(2) \lambda_{k}^{-1 / 2} L_{k}=$ $\sum_{j} u_{k j} L_{j}^{T}$ for some $\lambda_{k}>0$ and some unitary self-adjoint matrix $U=\left(u_{k j}\right)_{k, j \in \mathcal{J}}$. 
TheOREM 14. The $Q M S \mathcal{T}$ satisfies the $Q D B-\theta$ with respect to the generic, faithful and invariant state $\rho$ if and only if there exists a privileged $G K S L$ representation of $\mathcal{L}$ given as in Proposition 13 with positive constants $\gamma_{j l}^{ \pm}$fulfilling

$$
\rho_{j} \gamma_{j l}^{+}=\rho_{l} \gamma_{l j}^{-} \quad \text { for all } 1 \leq j<l \leq n-1 .
$$

Proof. Consider a privileged representation

$$
\begin{aligned}
\mathcal{L}(a) & =i[H, a]-\frac{1}{2} \sum_{k \in \mathcal{J}_{1}}\left(L_{k}^{*} L_{k} a-2 L_{k}^{*} a L_{k}+a L_{k}^{*} L_{k}\right) \\
& -\frac{1}{2} \sum_{j<l} \gamma_{j l}^{+}\left(E_{j}^{j} a-2 E_{j}^{l} a E_{l}^{j}+a E_{j}^{j}\right)-\frac{1}{2} \sum_{j<l} \gamma_{l j}^{-}\left(E_{l}^{l} a-2 E_{l}^{j} a E_{j}^{l}+a E_{l}^{l}\right),
\end{aligned}
$$

given as in Proposition 13 with $\gamma_{j l}^{ \pm}$satisfying 23 . Clearly we have $H=\bar{H}$. We recall that, by setting

$$
L_{j l}^{+}=\sqrt{\gamma_{j l}^{+}} E_{l}^{j}, \quad L_{j l}^{-}=\sqrt{\gamma_{l j}^{-}} E_{j}^{l}, \quad \lambda_{j l}=\rho_{l} \rho_{j}^{-1} \quad \text { for all } j<l,
$$

there holds

$$
\begin{aligned}
& \rho L_{k}=L_{k} \rho \\
& \text { for all } k \in \mathcal{J}_{1} \\
& \rho L_{j l}^{+}=\lambda_{j l} L_{j l}^{+} \rho, \quad \rho L_{j l}^{-}=\lambda_{j l}^{-1} L_{j l}^{-} \rho \quad \text { for all } j<l .
\end{aligned}
$$

Therefore, condition (2) of Theorem 4 can be rewritten as

$$
\left(\begin{array}{c|c|c}
\mathbb{1}_{\left|\mathcal{J}_{1}\right|} & 0 & 0 \\
\hline 0 & \Lambda & 0 \\
\hline 0 & 0 & \Lambda^{-1}
\end{array}\right)\left(\begin{array}{c}
L . \\
L_{.}^{+} \\
L_{\cdot}^{-}
\end{array}\right)=U\left(\begin{array}{c}
L^{T} \\
\left(L_{.}^{+}\right)^{T} \\
\left(L_{\cdot}^{-}\right)^{T}
\end{array}\right)
$$

for some unitary self-adjoint matrix $U=\left(u_{k j}\right)_{k, j \in \mathcal{J}}$, where $\mathbb{1}_{\left|\mathcal{J}_{1}\right|}$ denotes the $\left|\mathcal{J}_{1}\right| \times\left|\mathcal{J}_{1}\right|-$ identity matrix and we define

$$
\begin{gathered}
\Lambda=\operatorname{diag}\left(\lambda_{12}^{-1 / 2}, \ldots, \lambda_{1 n}^{-1 / 2}, \lambda_{23}^{-1 / 2}, \ldots, \lambda_{2 n}^{-1 / 2}, \ldots, \lambda_{n-1 n}^{-1 / 2}\right) \\
L .=\left(L_{1}, \ldots, L_{\left|\mathcal{J}_{1}\right|}\right)^{T}, \\
L^{ \pm}=\left(L_{12}^{ \pm}, \ldots, L_{1 n}^{ \pm}, L_{23}^{ \pm}, \ldots, L_{2 n}^{ \pm}, \ldots, L_{n-1 n}^{ \pm}\right)^{T} .
\end{gathered}
$$

Since $\left(L_{j l}^{+}\right)^{T}=\sqrt{\gamma_{j l}^{+}} E_{j}^{l}$ and $\left(L_{j l}^{-}\right)^{T}=\sqrt{\gamma_{l j}^{-}} E_{l}^{j}$ for every $j<l$, condition 29l implies $\gamma_{j l}^{+}=0$ if and only if $\gamma_{l j}^{-}=0$; otherwise we have

$$
\lambda_{j l}^{-1 / 2} L_{j l}^{+}=\sqrt{\frac{\rho_{j} \gamma_{j l}^{+}}{\rho_{l} \gamma_{l j}^{-}}}\left(L_{j l}^{-}\right)^{T}, \quad \lambda_{j l}^{1 / 2} L_{j l}^{-}=\sqrt{\frac{\rho_{l} \gamma_{l j}^{-}}{\rho_{j} \gamma_{j l}^{+}}}\left(L_{j l}^{+}\right)^{T}
$$

for all $(j, l) \in N:=\left\{(h, k): 1 \leq h<k \leq n\right.$ such that $\left.\gamma_{h k}^{+} \neq 0\right\}$.

As a consequence, since $L_{k}^{T}=L_{k}$ for all $k \in \mathcal{J}_{1}$, equation 290 holds if and only if

$$
U=\left(\begin{array}{c|c|c}
\mathbb{1}_{\left|\mathcal{J}_{1}\right|} & 0 & 0 \\
\hline 0 & 0 & U_{0} \\
\hline 0 & U_{0}^{-1} & 0
\end{array}\right)
$$

where

$$
U_{0}:=\operatorname{diag}\left(\sqrt{\frac{\rho_{1} \gamma_{12}^{+}}{\rho_{2} \gamma_{21}^{-}}}, \ldots, \sqrt{\frac{\rho_{1} \gamma_{1 n}^{+}}{\rho_{n} \gamma_{n 1}^{-}}}, \ldots, \sqrt{\frac{\rho_{n-1} \gamma_{n-1 n}^{+}}{\rho_{n} \gamma_{n n-1}^{-}}}\right),
$$


with the convention that terms corresponding to $(j, l) \notin N$ are set equal to 1 . Hence, $U$ is unitary and self-adjoint if and only if $U_{0}$ is unitary, i.e.

$$
\frac{\rho_{j}}{\rho_{l}} \frac{\gamma_{j l}^{+}}{\gamma_{l j}^{-}}=1 \quad \text { for all }(j, l) \in N .
$$

Finally, by condition 23 it is enough to have the previous equality only for $(j, l) \in N$ with $1 \leq j<l \leq n-1$. This is clearly equivalent to 28.

Analysing the proof of the previous theorem, we can immediately see that under the QDB- $\theta$ condition the matrix $U_{0}$ given by $(30)$ is the identity matrix, since the constants $\gamma_{j l}^{+}$and $\gamma_{l j}^{-}$satisfy $\rho_{j} \gamma_{j l}^{+}=\rho_{l} \gamma_{l j}^{-}$for all $j<l$ (by 28) and (23)). Therefore, we can rewrite Theorem 14 in the following way.

COROLlary 15. The $Q M S \mathcal{T}$ satisfies the $Q D B-\theta$ if and only if there exists a privileged representation of $\mathcal{L}$ given by operators

$$
\begin{array}{ll}
H=\sum_{j=1}^{n} h_{j} E_{j}^{j}, & h_{j} \in \mathbb{R} \quad \text { for all } j=1, \ldots, n, \\
L_{k}=\sum_{j=1}^{n} z_{k j} E_{j}^{j} \quad \text { for } k \in \mathcal{J}_{1} \subseteq\{1, \ldots, n-1\}, \\
L_{j l}^{+}=\sqrt{\gamma_{j l}^{+}} E_{l}^{j}, \quad L_{j l}^{-}=\sqrt{\rho_{j} \rho_{l}^{-1}}\left(L_{j l}^{+}\right)^{T} \quad \text { for all }(j, l) \in N,
\end{array}
$$

where $\gamma_{j l}^{+}$are positive constants for all $j<l, N=\left\{(j, l): j<l, \gamma_{j l}^{+} \neq 0\right\}$,

$$
z_{k n}=-\sum_{i=1}^{n-1} z_{k i} \rho_{i} \rho_{n}^{-1}
$$

and $\left\{\left(z_{k 1}, \ldots, z_{k n-1}\right): k \in \mathcal{J}_{1}\right\}$ is a set of linearly independent vectors in $\mathbb{C}^{n-1}$.

REMARK 16. Corollary 15 shows that the generator of a QMS satisfying the QDB- $\theta$ condition admits a privileged GKSL representation determined by

(a) $n-1$ real parameters $h_{j}$ for the Hamiltonian $H$, since $H$ is uniquely determined up to a real multiple of the identity operator;

(b) $n-1$ complex parameters $z_{k 1}, \ldots, z_{k n-1}$ for each $L_{k}$ with $k \in \mathcal{J}_{1}$, and $\left|\mathcal{J}_{1}\right| \leq n-1$;

(c) $n(n-1) / 2$ positive parameters $\left\{\gamma_{l j}^{+}: j<l\right\}$.

Moreover, two sets $\left\{z_{k}=\left(z_{k 1}, \ldots, z_{k n-1}\right): k \in \mathcal{J}_{1}\right\}$ and $\left\{z_{k}^{\prime}=\left(z_{k 1}^{\prime}, \ldots, z_{k n-1}^{\prime}\right): k \in \mathcal{J}_{1}\right\}$ of vectors in $\mathbb{C}^{n-1}$ lead to a privileged GKSL representation of the same generator if and only if there exists a unitary $U$ on $\mathbb{C}^{n-1}$ such that $U z_{k}=z_{k}^{\prime}$ for all $k=1, \ldots, n-1$. By the arguments of Remark 12 we remain then with $(n-1)^{2}$ free real parameters.

Thus the convex cone of generators of QMS satisfying the QDB- $\theta$ condition is a manifold with real dimension

$$
(n-1)+(n-1)^{2}+\frac{n(n-1)}{2}=\frac{3 n(n-1)}{2} .
$$

This, compared with the computation of Remark 12, shows that the QMS satisfying the SQDB- $\theta$ condition are much more numerous. 
Acknowledgments. The financial support of the MIUR PRIN 2007 project "Quantum Probability with Applications to Information Theory" is gratefully acknowledged.

\section{References}

[1] L. Accardi, K. Imafuku, Dynamical detailed balance and local KMS condition for nonequilibrium states, Internat. J. Modern Phys. B 18 (2004), 435-467.

[2] G. S. Agarwal, Open quantum Markovian systems and the microreversibility, Z. Physik 258 (1973), 409-422.

[3] R. Alicki, On the detailed balance condition for non-Hamiltonian systems, Rep. Math. Phys. 10 (1976), 249-258.

[4] F. Cipriani, Dirichlet forms and Markovian semigroups on standard forms of von Neumann algebras, J. Funct. Anal. 147 (1997), 259-300.

[5] J. Dereziński, R. Früboes, Fermi golden rule and open quantum systems, in: Open Quantum Systems III (Grenoble, 2003), Lecture Notes in Math. 1882, Springer, Berlin, 2006, $67-116$.

[6] F. Fagnola, R. Rebolledo, From classical to quantum entropy production, in: Quantum Probability and Infinite Dimensional Analysis (Hammamet, Tunisia, 2008), QP-PQ: Quantum Probab. White Noise Anal. 25, World Scientific, Hackensack, NJ, 2010, 245-261.

[7] F. Fagnola, V. Umanità, Generators of detailed balance quantum Markov semigroups Infin. Dimens. Anal. Quantum Probab. Relat. Top. 10 (2007), 335-363.

[8] F. Fagnola, V. Umanità, Detailed balance, time reversal and generators of quantum Markov semigroups (in Russian), Mat. Zametki 84 (2008), 108-116; English transl.: Math. Notes 84 (2008), 108-115.

[9] F. Fagnola, V. Umanità, On two quantum versions of the detailed balance condition, in: Noncommutative Harmonic Analysis with Applications to Probability II, Banach Center Publ. 89, Polish Acad. Sci., Inst. Math., Warsaw, 2010, 105-119.

[10] F. Fagnola, V. Umanità, Generators of KMS symmetric Markov semigroups on $\mathcal{B}(h)$ symmetry and quantum detailed balance, Comm. Math. Phys. 298 (2010), 523-547.

[11] S. Goldstein, J. M. Lindsay, Beurling-Deny condition for KMS-symmetric dynamical semigroups, C. R. Acad. Sci. Paris Sér. I Math. 317 (1993), 1053-1057.

[12] A. Kossakowski, A. Frigerio, V. Gorini, M. Verri, Quantum detailed balance and KMS condition, Comm. Math. Phys. 57 (1977), 97-110.

[13] W. A. Majewski, On the relationship between the reversibility of detailed balance conditions, Ann. Inst. H. Poincaré Sect. A (N.S.) 39 (1983), 45-54.

[14] W. A. Majewski, R. F. Streater, Detailed balance and quantum dynamical maps, J. Phys. A 31 (1998), 7981-7995.

[15] K. R. Parthasarathy, An Introduction to Quantum Stochastic Calculus, Monogr. Math. 85, Birkhäuser, Basel, 1992.

[16] P. Talkner, The failure of the quantum regression hypothesis, Ann. Physics 167 (1986), 390-436. 